\title{
Evidências psicológicas do surfe: efeitos terapêuticos e demandas assistenciais
}

\section{RESUMO}

O presente trabalho caracteriza-se como um estudo de revisão sistemática sobre pesquisas que tenham utilizado medidas psicológicas para avaliar atletas e praticantes de surfe. Foram utilizados os descritores "Surf" AND "Sport" e "Surf" AND "Psychology" e após aplicação dos critérios de exclusão, obteve-se uma amostra final de 28 artigos. Foram identificados estudos diversificados explorando aspectos psicológicos diferenciados nesse contexto, como questões de gênero, processos psicossociais, produção da subjetividade, efeitos terapêuticos e aspectos psicológicos concernentes ao universo da competição. Os resultados foram discutidos em categorias temáticas específicas, dentre as quais, destacam-se o estudo de processos psicológicos diretamente associados à prática do surfe; produções sobre o potencial terapêutico desencadeado pela prática do surfe; e também algumas evidências sobre efeitos adversos, principalmente no contexto competitivo. As evidências reforçaram a importância da assistência psicológica aos atletas de alto rendimento para promoção e manutenção da saúde mental, bem como para melhorias no desempenho esportivo. No lazer e em projetos específicos, o potencial terapêutico do surfe também se apresenta como um campo promissor.

PALAVRAS-CHAVE: Surfe; Psicologia; Saúde; Desempenho
Débora Matias de Souza

Graduanda em Psicologia e Bolsista de Iniciação Científica (PROPPI-UFF) Universidade Federal Fluminense, Campos dos Goytacazes, RJ, Brasil. deboramatiasousa@hotmail.com https://orcid.org/0000-0003-2144-501X

Monick Leonora Inês Kort Kamp Graduanda em Psicologia e Bolsista de Iniciação Científica (FAPERJ) Universidade Federal Fluminense, Campos dos Goytacazes, RJ, Brasil. kortkampmonick@gmail.com https://orcid.org/0000-0001-8019-6215

\section{Léo Barbosa Nepomuceno}

Doutor em Saúde Coletiva Universidade Federal do Ceará, Instituto de Educação Física e Esportes, Fortaleza, CE, Brasil.

leobnepomuceno@hotmail.com ㄴ https://orcid.org/0000-0002-7473-9460

Erick Francisco Quintas Conde Doutor em Neurociências

Universidade Federal Fluminense, Departamento de Psicologia, Campos dos Goytacazes, RJ, Brasil. psicoerick@yahoo.com.br https://orcid.org/0000-0002-7130-2888 


\title{
Psychological evidences of surf: therapeutic effects and assistance demands
}

\begin{abstract}
The present work is characterized as a systematic review of researches that have used psychological measures to evaluate surf athletes and practitioners. The descriptors used were "Surf" AND "Psychology" and "Surf" AND "Sport" and after applying inclusion and exclusion criteria, was obtained a final sample of 28 articles. Diversified studies were found exploring different psychological aspects in this field, such gender issues, psychosocial processes, subjectivity development, therapeutic effects and psychological aspects concerning the competitive context. The results were discussed in specific categories, as those directly associated with the surf practice; works about the therapeutic potential triggered by the surfing practice; and also some evidences about some adverse effects, especially in the competition context. The evidences reinforce the relevance of psychological assistance to high-performance athletes to promotion and maintenance of the mental health, as well to improvements in surf performance. In leisure activities and specific projects, the therapeutic potential of surfing also appears as a promising field.
\end{abstract}

KEYWORDS: Surf; Psychology; Health; Performance

\section{Evidencias psicológicas del surf: efectos terapéuticos y demandas de asistencia}

\section{RESUMEN}

El presente trabajo se caracteriza como un estudio de revisión sistemática sobre investigaciones que han utilizado medidas psicológicas para evaluar atletas y practicantes de surf. Se utilizaron de los descriptores "Surf" AND "Psychology" y "Surf" AND "sport", después aplicar los criterios de inclusión y exclusión, se tienes una muestra final de 28 artículos. Fueron encontrados estudios explorando diferentes aspectos psicológicos en este campo, como temas de género, procesos psicosociales, desarrollo de la subjetividad, efectos terapéuticos y aspectos psicológicos relacionados con el contexto competitivo. Los resultados fueron discutidos en categorías específicas, como los directamente relacionados con la práctica del surf; trabajos sobre el potencial terapéutico desencadenados por la práctica del surf; y también algunas evidencias sobre efectos adversos, especialmente en el contexto competitivo. Las evidencias refuerzan la relevancia de asistencia psicológica a los atletas de alto rendimiento para promover y mantener la salud mental, así como para mejorar el rendimiento del surf. En las actividades de ocio y proyectos específicos, el potencial terapéutico del surf también aparece como un campo prometedor.

PALABRAS-CLAVE: Surf; Psicología; Salud; Rendimiento 


\section{INTRODUÇÃO}

O surfe é uma modalidade esportiva com uma longa história que remonta a antigas formas de navegação e tradições desenvolvidas pelos povos originários do Peru e das ilhas da Polinésia. Esta modalidade desenvolve-se atualmente como uma prática de lazer e de competição, com ampla expansão pelo mundo (KAMPION; BROWN, 1998; BABOGHLUIAN, 2003; WARSHAW, 2010). A disseminação global da prática do surfe ocorre em função de vários motivos, como a busca de melhorar a saúde, a conexão com a natureza, por lazer e divertimento, superação de limites e motivos profissionais. Recentemente, a história do surfe sofreu um importante marco histórico, tendo sido incluída pelo Comitê Olímpico Internacional como modalidade-teste para os jogos Olímpicos do Japão, em 2020. Os recentes títulos mundiais obtidos pelos brasileiros Adriano de Souza, Gabriel Medina e Ítalo Ferreira também tem sido implicados com o aumento da popularidade da modalidade no Brasil (FERREIRA, 2018).

Revisões de literatura científica sobre o surfe, no tocante às questões socioculturais, destacam sua historiografia, desigualdades de gênero, interação entre surfistas e natureza, conflitos de sociabilidade derivados do localismo, relações entre esporte, mídia e religião (BRASIL; RAMOS; GODAS, 2013; PÉREZ-GUTIÉRREZ; COBO-CORRALES, 2020). No entanto, pouca produção científica sobre o surfe tem sido observada na área da Psicologia do esporte, especialmente no tocante aos aspectos psicológicos implicados diretamente à prática do surfe, os modos como seus praticantes se apropriam da modalidade, suas motivações e as implicações da prática para a saúde, entre outros.

Adicionalmente, o número de programas que utilizam o surfe como dispositivo terapêutico vem aumentando consideravelmente nos últimos anos. Segundo Benninger e colaboradores (2020), os primeiros programas terapêuticos tendo o surfe como intervenção principal, surgiram na década de 1990, tendo esse número aumentado para mais de 50 programas de surfe terapia espalhados pelo mundo em 2020. Tais programas terapêuticos tem sido destinados ao atendimento de uma população bem diversificada, sejam jovens com deficiência, pessoas com transtornos psicológicos, TEA e veteranos de guerra com TEPT. Em termos de publicação científica, Benninger e colaboradores (2020) indicaram que a primeira evidência científica sobre propriedades terapêuticas do surfe, só foi publicada em uma revista com revisão por pares no ano de 2010 (MORGAN, 2010) e que, apenas recentemente, foram publicados alguns poucos ensaios de controle randomizados (WALTTERS et al, 2019a, 2019b). Para os autores, existe ainda uma escassez de pesquisas sobre as 
práticas baseadas em evidências com surfe terapia que apóiem o impacto global das intervenções existentes.

Considerando as demandas para ampliação do conhecimento científico associado ao surfe em suas diferentes dimensões, presente pesquisa se direcionada ao levantamento de produções originais pertinentes a esse universo, com o objetivo de analisar como os processos psicológicos têm sido estudados neste contexto.

\section{MÉTODOS}

A metodologia foi elaborada com base no modelo Preferred Reporting Items for Systematic Reviews and Meta-Analyses (PRISMA) (MOHER et al., 2015). As consultas foram feitas nas plataformas Web of knowledge e Pubmed (US National Library of Medicine) em agosto de 2019, com os descritores "Surf" AND "Psychology" e "Surf” AND "Sport”. Para inclusão de trabalhos no estudo, foram adotados os seguintes critérios: trabalhos publicados nos últimos dez anos que antecederam à consulta; trabalhos em conformidade com o tema surfe e psicologia ou surfe e esporte e que envolvam diretamente os construtos principais aqui tratados. Estabelecemos o critério de incluir apenas os trabalhos originais que avaliassem aspectos psicológicos na prática do surfe. Além deste, consideramos apenas os trabalhos disponíveis em formato completo nas bases de dados citadas; trabalhos escritos em língua inglesa ou que contenham um resumo em inglês. Estudos teóricos, meta-análises e revisões sistemáticas foram também excluídos da revisão. Para a extração dos dados na amostra final foram selecionadas as seguintes informações: ano da publicação, autores, amostra, aspectos psicológicos avaliados e principais resultados encontrados.

O processo de seleção dos trabalhos seguiu as etapas indicadas: 1) busca pelos descritores nas bases de dados; 2) exclusão dos trabalhos publicados há mais de dez anos; 3) exclusão dos trabalhos duplicados; 4) leitura dos resumos para verificação da adequação aos temas e critérios; 5) busca nas bases de dados virtuais pelo trabalho completo; 6) leitura completa e análise qualitativa dos artigos selecionados para amostra final.

Após a delimitação do conjunto de artigos selecionados, o mesmo foi submetido a processo de análise qualitativa de seus conteúdos, onde foram apreendidos os principais resultados e detalhes metodológicos de cada trabalho. Como parte da análise qualitativa dos artigos, foram construídas as categorias temáticas para sistematizar a discussão e interpretação dos mesmos: 1) aspectos psicológicos gerais pertinentes à prática; 2) implicações do surfe ao desenvolvimento humano; 3) efeitos terapêuticos desencadeados pelo surfe; e 4) efeitos negativos associados à modalidade. 
A análise adotou parâmetros prescritos pelo modelo da Análise Temática Reflexiva (BRAUN; CLARKE, 2006; SOUZA, 2019). Mais especificamente, este modelo de análise pode ser caracterizado como um procedimento que possui o propósito de auxiliar a identificar, interpretar e caracterizar padrões (ou temas) a partir de uma perspectiva qualitativa (BRAUN; CLARKE, 2006; SOUZA, 2019). Dentre os modelos de Análise Temática, utilizamos a abordagem Reflexiva, que tem como princípio a codificação fluida e flexível de características específicas da amostra (como classificação conceitual, tipo de incidência, cronologia, entre outros aspectos) na interpretação dos dados, priorizando a identificação de temas, padrões ou classes específicas de significação à quantificação ou à acurácia de instrumentos de medidas. O presente estudo utilizou princípios da Análise Temática Reflexiva para caracterização e discussão dos resultados de acordo com os temas estudados pelos artigos encontrados na revisão sistemática.

\section{RESULTADOS}

Na revisão realizada na plataforma PubMed com os descritores "Surf" AND "Psychology", verificamos inicialmente a ocorrência de 51 artigos. Porém, ao aplicarmos os critérios de inclusão e exclusão, a amostra final foi composta por apenas 1 artigo (PITTSINGER, 2017). Ao utilizarmos os mesmos descritores na plataforma Web of Science obteve-se inicialmente uma amostra de 31 artigos, porém ao aplicarmos os critérios de inclusão e exclusão somente 4 artigos estiveram aptos a compor o banco de dados (PARTINGTON et al., 2009; BRYMER; OADES, 2009; WIERSMA, 2014; PITTSINGER et al., 2017; CORREIA; ROSADO, 2019). Assim, utilizando os referidos descritores, tivemos o total de 5 artigos que atenderam aos critérios estipulados (Tabela 1, em apêndice).

Devido ao pequeno número de artigos encontrados na pesquisa com os descritores "Surf" AND "Psychology", também foram utilizados os termos "Surf" AND "Sport" em uma consulta subsequente, adotando o critério adicional de que o estudo envolvesse alguma medida ou verificasse algum aspecto psicológico. Ao pesquisarmos com essa configuração na PudMed, tivemos como resultado 140 artigos. Ao aplicarmos o filtro dos últimos dez anos de publicações, tivemos um resultado de 88 artigos e, após aplicar todos os critérios já aqui descritos, tivemos uma amostra final de quatro artigos. Usando os mesmos descritores na plataforma Web of Science e após a aplicação de todos os critérios, o banco de dados se estabeleceu com 23 artigos (tabela 2, em apêndice). Considerando as duas pesquisas realizadas, obteve-se uma amostra final de 28 artigos que atenderam aos critérios desta revisão. 
Nessa perspectiva, para facilitar a apresentação e discussão dos resultados, tal como proposto por Souza e colaboradores (2010), a amostra de artigos foi qualitativamente avaliada quanto aos objetos de estudo e posteriormente, os resultados foram agrupados em categorias interpretativas tal como preconizado pelos modelos de análise temática reflexiva (BRAUN; CLARKE, 2006; SOUZA, 2019).

\section{DISCUSSÃO}

É importante ressaltar que o banco de dados composto por 28 artigos que tenham utilizado medidas psicológicas no contexto do surfe, só foi possível ser estabelecido ampliando o escopo de busca para além das palavras-chave "Surf" e "Psychology". Apesar das contribuições consistentes da Psicologia do Esporte nos últimos anos (CONDE et al., 2019), a construção do conhecimento científico inerente aos processos psicológicos no surfe tem sido feita em perspectiva interdisciplinar. Sendo assim, a maioria dos trabalhos só foi acessada com a utilização os termos "Surf" AND "Sport".

Dentre os artigos encontrados, foi possível observar uma grande diversidade de processos e fenômenos psicológicos investigados. A análise permitiu a identificação de quatro categorias temáticas específicas. Em uma dessas categorias, foram agrupados os artigos que apresentavam implicações ou características inerentes ao campo da psicologia do desenvolvimento, seja através de perspectivas comparativas entre atletas experientes e novatos (PITTSINGER et al., 2017), sobre desenvolvimento da subjetividade, da corporeidade, da identidade e da dimensão biopsicossocial.

Muitos outros estudos se aplicaram ao estudo das implicações do surfe na promoção da saúde mental e de outros efeitos terapêuticos. Com o crescente número de artigos e evidências científicas sobre os efeitos terapêuticos associados ao surfe, o emprego do termo Surfe terapia tem sido utilizado pela International Surf Therapy Organization (ISTO) para caracterizar um método de intervenção que combina a instrução para o desenvolvimento no surf com atividades estruturadas para promoção de bem-estar psicológico, psicossocial, de saúde física e mental. Assim, alguns dos artigos originais encontrados em nossa revisão que estudaram efeitos terapêuticos foram agrupados em uma subseção intitulada "O potencial terapêutico do surfe". Nesta temática apontaremos evidências que sugerem que programas terapêuticos através do surfe são efetivos para auxiliar na melhoria da qualidade de vida e ajudar na promoção da saúde mental em quadros clínicos específicos, como o Transtorno de Estresse Pós-Traumático (TEPT), Transtorno do Espectro 
Autista (TEA), quadros de ansiedade e depressão e também como elemento de suporte e desenvolvimento social.

Contudo, também foram verificados relatos científicos de aspectos negativos implicados ao universo do surfe, como a dependência pela prática, riscos físicos, e fatores estressores provenientes do contexto de competição. Os artigos que não se enquadraram nas categorias acima identificadas, foram agrupados em uma categoria temática mais geral para discussão. Neste âmbito, perspectivas de estudo com metodologias variadas exploraram processos mais gerais associados à prática do surfe. Assim, optamos por discutir inicialmente alguns desses estudos, como evidências de processos psicológicos mais amplos e variados no contexto do surfe.

\section{Aspectos psicológicos gerais pertinentes à prática do surfe}

Pittsinger e colaboradores (2017) estudaram o efeito de 30 minutos de prática do surfe sobre o Afeto induzido pelo exercício, utilizando a Escala de Afetividade da Atividade Física (Physical Activity Affect Scale, PAAS). Os resultados apontaram que o afeto positivo e percepções de tranquilidade aumentaram significativamente após a prática do surfe, enquanto o afeto negativo e a percepção de fadiga diminuíram significativamente.

Wiersma (2014) indicou a existência de variações emocionais em seis fases específicas do surfe competitivo de ondas grandes. Os entrevistados relataram variedade e oscilações de estados mentais. A ansiedade foi amplamente identificada no momento que antecede a entrada dos surfistas no mar, com dúvidas frequentes acerca da sua preparação física e mental. Já, o medo, foi comumente relatado e associado ao momento antes de ficarem em pé na prancha para pegar a onda. Reforçaram que sentimentos de confiança e de comprometimento são também necessários para entrar no mar e surfar. Já na fase de wipe-out (queda da onda), os surfistas relataram estresse, desespero, euforia e esgotamento mental, pelo alto risco de lesões e até mesmo, de vida.

Brasil e colaboradores (2016) investigaram ações pedagógicas para o ensino do surfe, objetivando compreender quais as melhores ações para que o aprendiz possa ter pleno desenvolvimento na modalidade. Os autores sugeriram que as intervenções pelos treinadores e instrutores devem atender especificidades de cada aluno e do ambiente. $\mathrm{O}$ estudo também indicou que a relação subjetiva que o aprendiz estabelece com a atividade e com o ambiente é crucial para adesão e desenvolvimento no surfe. Ressalta-se a relevância do estudo em atentar-se à questão primordial que é a iniciação à modalidade. 


\section{Implicações do surfe ao desenvolvimento biopsicossocial}

Alguns estudos encontrados apresentaram implicações ao campo da psicologia do desenvolvimento, indicando efeitos estruturantes do surfe em funções psíquicas, na formação da identidade e da subjetividade. O estudo de Furley e Dorr (2016) investigou processos de tomada de decisão em surfistas, considerando como variável independente, o quesito "experiência". Os voluntários foram divididos em três grupos conforme experiência no esporte (grupo controle, surfistas principiantes, e surfistas com mais de dez anos de prática). Os pesquisadores fizeram uma simulação virtual das ondas se aproximando e os participantes deveriam indicar pressionando uma tecla indicativa se a onda seria surfável ou não. Como resultados principais, os autores apontaram que os surfistas com maior experiência apresentaram melhor acurácia, fazendo melhor distinção das ondas surfáveis das não surfáveis. Este estudo forneceu uma das primeiras evidências de que surfistas altamente experientes possuem vantagens cognitivas quando comparados a surfistas menos experientes ou a um grupo controle formado por pessoas que não surfam.

Algumas pesquisas consideram que o surfe pode servir também como aspecto estruturante da identidade e da subjetividade humana (SCHIMID, 2019; ROY, 2014; et al., 2019; WHEATON, 2017; WHEATON et al., 2017; WHEATON, 2019). Roy (2014) entrevistou 35 mulheres com idades entre 12 e 65 anos, que indicaram a existência de demandas emocionais importantes em torna-se surfista, considerando que a capacidade de surfar está diretamente ligada ao fato de se sentirem confortáveis para essa prática. Algumas mulheres entrevistadas relataram estratégias específicas para competir com homens pelo domínio de espaço e das ondas e outras, por exemplo, escolhem momentos do dia menos concorridos para surfar. Neste estudo, a maioria das entrevistadas expressou que a prática possibilita sentimentos que ajudam a estabelecer a identidade e a subjetividade pessoal.

Rynne (2016) buscou investigar as contribuições do surfe como fator de desenvolvimento físico e social nas vidas de adolescentes indígenas. Neste estudo, foram avaliados 54 participantes com variados níveis de envolvimento com a modalidade. Destes, 23 eram jovens indígenas australianos que participavam de um programa de surfe. Os resultados apontam que, dentre as pessoas envolvidas no projeto, a maioria adotava uma visão holística sobre a prática, compreendendo que esta propiciava também a reconexão com o mar e benefícios para toda a comunidade. Os voluntários relatam ainda que a prática tem sido um importante pilar para o repasse da cultura, propiciando a (re) conexão com a natureza, com o oceano, o desenvolvimento de conhecimento específico para o surfe e para interação com o oceano, (re) estabelecimento de laços sociais e comunitários. 
O surfe também parece ter implicações ao desenvolvimento da corporeidade (BUSH, 2016). A construção da corporeidade feminina foi objeto de estudo na pesquisa de Bush (2016), que objetivou examinar como a cultura do surfe influenciou no desenvolvimento do estilo de vida e da identidade de mulheres na costa leste dos Estados Unidos. A pesquisadora verificou que a prática propiciou sentimentos de felicidade, melhorias em capacidades físicas e mentais. O surfe foi considerado por muitas como uma forma de terapia, utilizada como estratégia para superar ou lidar com adversidades emocionais.

Os estudos de Wheaton e colaboradores (WHEATON, 2017; WHEATON et al., 2017; WHEATON, 2019) implicam a prática do surfe na formação e no desenvolvimento da identidade em jovens (WHEATON, 2017) e também em adultos e pessoas idosas (WHEATON et al., 2017; WHEATON, 2019), ressaltando implicações ao desenvolvimento social e comunitário (WHEATON, 2017). Outras evidências indicaram que esportes radicais, como o surfe podem desenvolver também valores específicos como a humildade e a coragem a partir da prática contínua (BRYMER; OADES, 2016).

\section{O potencial terapêutico do surfe}

Em nossos resultados foram identificados vários estudos que reforçam a proposta de que o surfe deve ser considerado uma atividade terapêutica, capaz de auxiliar na melhoria sintomatológica em diversos quadros clínicos. O declínio da ansiedade, do estresse e de sintomas depressivos a partir da iniciação no surfe foram aspectos observados em algumas das pesquisas encontradas em nossa revisão (SCHMID et al., 2019; AMRHEIN et al., 2016; ROGERS et al., 2014; THORPE, 2015; SCHVIRTZ, 2018). Amrhein e colaboradores (2016) investigaram se surfistas relatariam menos sintomas de depressão e ansiedade do que a amostra normativa. Os resultados demonstraram que surfistas apresentam relatos acerca da espiritualidade e demonstram maior qualidade de vida, menores índices de depressão e de ansiedade. A prática do surfe também se mostrou eficaz na diminuição de sintomas do Transtorno de Estresse Pós Traumático (TEPT) como mostraram Caddick e colaboradores (2015a; 2015b), Caddick e Smith (2017), Rogers (2014), Caddick (2015) e Schvirtz (2018).

Outras implicações terapêuticas consideram que a prática do surfe pode desencadear aumento na autoconfiança (SCHIMID, 2019; MARSHALL, 2019; ROGERS, 2014; WIERSMA, 2014), mais sentimentos de felicidade (GOLDFREY, 2015; BUSH, 2016), maior sensação de liberdade (CADDICK; SMITH, 2017; CADDICK et al.,2015b) e aumento da resiliência (THORPE, 2015; MARSHALL, 2019). A sensação de bem-estar advinda da prática do surfe também foi 
evidenciada em alguns dos estudos identificados (MARSHALL, 2019; WHEATON et al., 2017; WHEATON, 2019; OLIVE, 2015; BEAUMONT; BROWN, 2015; BRASIL et al., 2016; CADDICK; SMITH, 2017; CADDICK et al., 2015b; SCHVIRTZ, 2018), aparecendo em aproximadamente $29 \%$ dos artigos inclusos.

Outros trabalhos indicaram efeitos positivos do surfe na qualidade das interações sociais (GODFREY, 2015; WHEATON et al., 2017; WHEATON, 2019; RYNNE, 2016; BEAUMONT, 2015; CADDICK et al., 2015a; THORPE, 2015). Neste quesito, o surfe aparece como aspecto que restabelece laços e afetividades, com implicações positivas também na economia de determinadas comunidades (BEAUMONT; BROWN, 2015; RYNNE, 2016).

Outros efeitos positivos advindos da prática do surfe foram o aumento do afeto positivo e da tranquilidade (PITTSENGER, 2017); melhoria de habilidades cognitivo-perceptivas (FURLEY; DORR, 2016); adequação funcional do comportamento (GOLDFREY, 2015); ampliação de sentimentos de empoderamento e autonomia (SCHIMID, 2019; MARSHALL, 2019).

Schmid e colaboradores (2019) investigaram os efeitos psicológicos decorrentes da prática do surfe em pessoas com deficiências variadas inseridos em um programa de reabilitação no Havaí conhecido como AccesSurf. Tal instituição tem por finalidade a capacitação de pessoas com deficiência para realizar natação e surfe adaptado. Para o estudo, foram recrutados participantes com mais de seis meses no programa. Os resultados apontaram melhorias nas capacidades físicas, mentais e sociais após a inserção no projeto. Descreveram o desenvolvimento de sentimentos de empoderamento, maior independência e que o surfe influenciou na reconstrução da identidade pessoal. Os participantes relataram declínio da ansiedade, do estresse e aumento da confiança. Descreveram também que sentiram uma melhoria significativa na qualidade dos relacionamentos interpessoais.

Godfrey, Devine e Taylor (2015) avaliaram 114 jovens encaminhados pelas redes de saúde, educação e assistência social, com diferentes queixas relacionadas à saúde mental ou exclusão social, ao projeto intitulado "Wave Project", que possui como proposta a intervenção psicossocial através da iniciação ao surfe, buscando promover saúde e bem-estar. O estudo utilizou a Escala de Bem-Estar de Crianças de Stirling (SCWBS) e analisou também relatos dos pais. Como resultados, os pesquisadores reportaram que $96 \%$ dos participantes sentiram mudanças positivas em suas vidas, 98\% se declararam mais felizes e que se divertiam bastante durante a prática do surfe (99\%). Relataram também que fizeram mais amigos (89\%) e que se sentiram mais aptos para as demandas do cotidiano (87\%). Sobre as medidas de bem-estar, as análises estatísticas demonstraram aumento significativo na pontuação da referida escala após a inserção nas atividades de surfe. O relato dos 
pais corroborou com os resultados encontrados com os participantes, reforçando o achado de que o impacto do projeto foi extremante positivo para a vida pessoal e familiar.

Marshall e colaboradores (2019), também estudaram efeitos terapêuticos associados ao Wave Project. Nesse estudo, indicaram que o surfe foi capaz de afetar indicadores de autonomia, bem-estar, confiança, de proporcionar melhorias na saúde e de ampliar a resiliência, além de melhorar os laços sociais dos praticantes. Segundo os pesquisadores, os resultados advêm da combinação da prática do surfe com o ambiente proporcionado pelo projeto, pois, segundo os entrevistados, o ambiente e as vivências psicossociais são variáveis que propiciam alívio de seus problemas cotidianos, diminuindo sintomas de ansiedade e depressão.

Rogers (2014) estudou implicações do surfe como coadjuvante ao tratamento de TEPT e depressão, entrevistando 11 veteranos de uma ação militar realizada no Iraque com o diagnóstico de TEPT, transtorno depressivo maior ou ambos. Os participantes relataram melhora significativa nos sintomas de TEPT e depressão, aumento da confiança e o desenvolvimento de habilidades psicológicas específicas. Caddick e colaboradores (2015) também investigaram como o surfe pode auxiliar veteranos de guerra diagnosticados com transtorno de estresse pós-traumático (TEPT), tendo identificado a diminuição de pensamentos disfuncionais e o desaparecimento de pensamentos suicidas. O surfe também aumentou felicidade, sentimentos de liberdade e propiciou melhorias na esfera social.

Thorpe (2015) verificou que modalidades de ação, incluindo o surfe, ajudaram seus praticantes a recriarem rotinas e imaginarem os espaços para além da destruição desencadeada por um terremoto em Christchurch (Nova Zelândia), em 2011. Os participantes afirmaram que os esportes ajudaram a vislumbrar novas possibilidades. Foi constatada a diminuição no estresse, na ansiedade e da tensão e que, em períodos de calamidade, a prática se tornou fundamental para que os entrevistados se tornassem mais resilientes e pudessem se recuperar.

O estudo realizado por Schvirtz e colaboradores (2018) se configura como relato de experiência de um surfista de 35 anos que sofreu um ataque de tubarão e teve uma amputação supracondilar do fêmur. Os pesquisadores relataram que o paciente se queixava de não conseguir praticar o surfe após a amputação e para a resolução de tal problema, foi desenvolvida uma prótese que permitia mais mobilidade e equilíbrio na prancha. Após a retomada das atividades, o participante passou a indicar que se sentia menos ansioso e também com menos dores neuropáticas. Em conclusão, Schvirtz e colaboradores (2018), afirmaram que essa atividade provavelmente pode auxiliar na reabilitação de amputados através da promoção de uma maior qualidade de vida e de bem-estar. 
Como pode ser observado, existem vários relatos científicos sobre efeitos positivos do surfe na saúde mental. Deve-se, portanto, considerar a prática dessa modalidade como alternativa terapêutica complementar, mas que requer de estudos mais aprofundados para uma maior compreensão de seus efeitos terapêuticos. Tais aplicações terapêuticas devem considerar também alguns efeitos negativos associados à prática e descritos abaixo.

\section{Efeitos negativos associados ao surfe}

Apesar de terem sido verificadas evidências sobre efeitos terapêuticos associados ao surfe, deve-se também considerar a pesquisa de Burgess e colaboradores (2018), sobre lesões e indicadores de saúde. O referido estudo demonstrou que existe uma alta incidência de lesões nas regiões lombar, nos pés, no joelho e tornozelo. Os tipos mais frequentes de lesão foram escoriação e laceração. Embora a ocorrência de lesões físicas seja alta nesse meio, foram verificados que os escores médios do componente saúde física e mental do SF-12 foram maiores do que a norma da população.

Partington e colaboradores (2009) também estudaram alguns efeitos negativos associados ao surfe, como o próprio nome do artigo sugere "The dark side of flow...". Partindo da hipótese de que o surfe poderia também causar uma espécie de dependência psicológica, os pesquisadores entrevistaram uma amostra de 15 renomados surfistas de ondas grandes, com uma entrevista semiestruturada. Como principais resultados, os autores indicaram que, apesar de inúmeros benefícios relatados, também foram relatadas evidências de que a prática pode se tornar algo vicioso. De acordo com os relatos, afirmativas de que precisavam experimentar sempre mais da experiência propiciada pelo surfe de ondas grandes, se assemelham a características de dependência psicológica. Contudo, os autores enfatizaram que somente alguns surfistas identificaram sinais de dependência, uma vez que colocaram a prática como prioridade, inclusive acima da própria vida, quando optaram a procurar sempre por ondas ainda mais perigosas. Tal estudo incita a reflexão sobre a centralidade que o surfe pode assumir no estilo de vida de praticantes que, quando em casos extremos, pode significar exposição a situações de risco, desencadear síndromes como Burnout, compulsão ou treinamentos excessivos, ampliando o risco de lesão relacionado à modalidade.

Outras evidências sobre efeitos negativos para quem usa o surfe como atividade laboral, são reportadas no trabalho de Evers (2019) que, através de entrevistas com 11 surfistas patrocinados para exposição midiática, indicou que a percepção de felicidade e bem estar no surfe não pode ser generalizada. Em sua pesquisa, alguns surfistas associaram características negativas ao trabalho midiático requisitado para divulgação do freesurf e que também são inerentes ao contexto 
competitivo. Este tem sido retratado também como sendo um ambiente hostil, que pode desencadear ansiedade e sensações de vulnerabilidade. O Freesurf é um termo utilizado para quem pratica o surfe como estilo de vida dissociado das competições.

Além dos estudos de Evers (2019), Correia e Rosado (2019) demonstraram que no ambiente de competição, sintomas de ansiedade e sentimentos de vulnerabilidade são realmente comuns. Este achado se opõe ao que foi identificado nas pesquisas de Schmid (2017), Amrhein (2016), Rogers (2014), Thorpe (2016) e Schvirtz (2018), que demonstram que praticar surfe pode diminuir os índices de ansiedade e de outros sintomas psicológicos. Possivelmente, tal diferença se deva ao fator "competição", que deve influenciar os estados psíquicos gerando mais ansiedade e tensão. Ainda assim, Wheaton (2017), reforça a tese de que pelo fato de o surfe ser um esporte individual e o fato do sujeito depender apenas de si mesmo, pode fazer com que se observe menor de ansiedade e estresse nas competições de surfe.

\section{CONCLUSÕES}

O surfe tem sido caracterizado como um esporte com características psicomotoras peculiares e uma significativa história sociocultural, que expressa uma rica relação do homem com a natureza, especialmente com o mar e com as ondas. Nossa revisão possibilita a compreensão de que o surf tem sido utilizado em perspectivas diversas e com objetivos variados, tal como para fins de lazer, como estilo de vida, inclusão social ou para reabilitação física e mental, bem como fonte de renda de empresas, atletas, instrutores e profissionais do esporte.

Os resultados iniciais nos identificaram a necessidade da realização de mais estudos que estudem de forma mais aprofundada os correlatos psicofisiológicos envolvidos na prática do surfe, bem como aspectos sociais envolvidos. Nossa revisão indicou que ainda são muitas as lacunas a serem exploradas, bem como a diversidade de temas a serem investigados. Acredita-se que o desenvolvimento de novas pesquisas e práticas em Psicologia do Esporte relacionadas ao surfe possam se desenvolver a partir das categorias temáticas identificadas neste estudo, já que compilam as referências científicas para subsidiar o desenvolvimento teórico, de protocolos de investigação e intervenção.

Cabe destacar o potencial terapêutico da prática da modalidade, que já tem sido explorado em diferentes estudos e aos poucos, se convertem em ações de extensão e intervenção espelhadas pelo mundo em diferentes projetos terapêuticos e de desenvolvimento psicossocial. No Brasil, são várias as iniciativas, como no projeto Onda Azul (SC), Surf4Mind (UFRJ) e no projeto sUrFF 
terapia (UFF). Os indícios de evolução terapêutica encontrados na reabilitação em quadros neurológicos e psicológicos devem ser considerados como estratégia de promoção de saúde através da iniciação esportiva nessa modalidade. Tais indicadores podem estimular o desenvolvimento de políticas públicas para acesso e como modalidade complementar por parte de populações clínicas, grupos de risco e como opção recreativa para a população em geral.

No contexto do surfe de alto rendimento, acredita-se que as produções científicas devem ser ampliadas significativamente em função do surfe ter sido indicado como modalidade teste nos jogos Olímpicos. Nesta temática, observamos produções que tenham indicado ocorrências de eventos negativos, como a experimentação da ansiedade competitiva, fatores tensionais, hostilidade e alterações de humor em competidores, reações de medo e insegurança em condições extremas, bem como desgaste e fadiga mental. Ainda que tais aspectos não tenham sido reportados com consistência, tais achados poderão auxiliar atletas de alto rendimento e suas respectivas comissões técnicas a compreenderem melhor as principais demandas de preparação psicológica para competições e para o surfe em condições extremas. Além do conhecimento, tais indicadores também reforçam a necessidade de suporte emocional aos atletas de alto rendimento dessa modalidade. Espera-se que futuros estudos nesse contexto também possam verificar a influência dos diferentes estados mentais no desempenho esportivo, bem como as implicações emocionais e cognitivas ao desempenho no surfe de alto rendimento. Acredita-se que a condição neo-olímpica desta modalidade possa estimular a produção científica não apenas no alto rendimento, mas também concernente às suas implicações aos campos da reabilitação, educação (e desenvolvimento), saúde, competição e lazer.

Como pode ser observado, existem demandas e potenciais de pesquisa muito promissores a serem explorados acerca dos efeitos da prática do surfe e sobre processos psicológicos inerentes à performance de surfistas competidores. A presente revisão sistemática contribui ao desenvolvimento científico da Psicologia do Esporte no contexto do surfe, compilando os principais estudos realizados até o momento e indicando, ao menos, quatro campos temáticos de desenvolvimento científico nesta articulação.

\section{REFERÊNCIAS}

AMRHEIN, Michael; ARKHOFF, Harald; HEIBY, Elaine. Spirituality, depression, and anxiety among ocean surfers. Journal of Clinical Sport Psychology, v. 10, n. 2, p. 155-171, 2016. doi: https://dx.doi.org/10.1123/jesp.2015-0016

BABOGHLUIAN, Marcelo. Surfe - esporte, estilo de vida. In: RUBIO, Katia. Psicologia do Esporte Aplicada. São Paulo: Casa do psicólogo, 2003. 
BEAUMONT, Emily; BROWN, David. 'Once a Local Surfer, Always a Local Surfer': Local Surfing Careers in a Southwest English Village. Leisure Sciences, v. 37, n. 1, p. 68-86, 2015. doi: https://dx.doi.org/10.1080/01490400.2014.952462

BENNINGER, Elizabeth; CURTIS, Chloe; SARKISIAN, Gregor.; ROGERS, Carly; BENDER, Kailey; COMER, Megan. Surf Therapy: A Scoping Review of the Qualitative and Quantitative Research Evidence Surf Therapy: A Scoping Review of the Qualitative and Quantitative Research Evidence. Global Journal of Community Psychology Practice, v. 11, p.1-26, 2020.

BRASIL, Vinicius; RAMOS, Valmor; DE SOUZA, Jeferson; BARROS, Thais; DO NASCIMENTO, Juarez. The pedagogical actions for the surf coach intervention. Movimento, v. 22, n.2, p. 403-416, 2016.

BRASIL, Vinícius Zeilmann; RAMOS, Valmor; GODA, Ciro. A produção científica sobre surf: uma análise a partir das publicações entre 2000-2011. Pensar a Prática, v. 16, n. 3, p. 619-655, 2013. doi: https://dx.doi.org/10.5216/rpp.v16i3.19466

BRYMER, Eric; OADES, Lyndsay. Extreme sports: A positive transformation in courage and humility. Journal of humanistic psychology, v. 49, n.1, p. 114-126, 2009. doi: https://dx.doi.org/10.1177/0022167808326199

BURGESS, Andrew; SWAIN, Michael; LYSTAD, Reidar. An Australian survey on health and injuries in adult competitive surfing. The Journal of sports medicine and physical fitness, v. 59, n. 3, p.462-468, 2019. doi: https://dx.doi.org/10.23736/S0022-4707.18.08381-0

BUSH, Lee. Creating our own lineup: Identities and shared cultural norms of surfing women in a US East Coast community. Journal of Contemporary Ethnography, v. 45 n. 3, p. 290-318, 2016. doi: https://dx.doi.org/10.1177\%2F0891241614556346

CADDICK, Nick; Smith, Brett. Combat surfers: A narrative study of veterans, surfing, and war trauma. Movimento, v. 23, n. 1, p. 25-38, 2017. doi: https://dx.doi.org/ 10.22456/1982-8918.71264

CADDICK, Nick; Smith, Brett; PHOENIX, Cassandra. Male combat veterans' narratives of PTSD, masculinity, and health. Sociology of health \& illness, v. 37, n. 1, p. 97-111, 2015a. doi: https://dx.doi.org/ $\underline{10.1111 / 1467-9566.12183}$

CADDICK, Nick; SMITH, Brett; PHOENIX, Cassandra. The effects of surfing and the natural environment on the well-being of combat veterans. Qualitative health research, v. 25, n. 1, p. 76-86, 2015b. doi: https://dx.doi.org/ 10.1177/1049732314549477

CONDE, Erick Francisco Quintas; FILGUEIRAS, Alberto; ANGELO, Luciana; PEREIRA, Adriana; CARVALHO, Cristianne Almeida. Psicologia do Esporte e do Exercício: Modelos teóricos, pesquisa e intervenção. 1. ed. - São Paulo: Pasavento, 2019.

CORREIA, Marco; ROSADO, António. Anxiety in Athletes: Gender and Type of Sport Differences. International Journal of Psychological Research, v. 12, n. 1, p. 9-17, 2019. doi: http://dx.doi.org/10.21500/20112084.3552

DIAS, Cléber; SOARES, Rafael Fortes; MELO, Vitor Andrade. Sobre as Ondas: surfe, juventude e cultura no Rio de Janeiro dos anos 1960. Estudos Históricos. Rio de Janeiro, v. 25, n. 49, p. 112-128, jan-jun. 2012. Disponível em: http://bibliotecadigital.fgv.br/ojs/index.php/reh/article/view/3669/2834. Acesso em: 20 maio 2018.

EVERS, Clífton. Westly. The gendered emotional labor of male professional 'freesurfers' digital media work. Sport in Society, v. 22, n. 10, p. 1691-1706, 2019. doi:

https://dx.doi.org/10.1080/17430437.2018.1441009 
FERREIRA, Ana Carolina. O surf e a tv aberta: uma análise midiática dos anos de 2014 e 2015. 2018. 52 f. Monografia (Bacharelado em Educação Física) - Departamento Acadêmico de Educação Física, Universidade Tecnológica Federal do Paraná, Curitiba, 2018.

FURLEY, Philipi; DÖRR, Jannic. "Eddie would (n't) go!" perceptual-cognitive expertise in surfing. Psychology of Sport and Exercise, v. 22, p. 66-71, 2016. doi:

https://doi.org/10.1016/j.psychsport.2015.06.008

GODFREY, Cath; DEVINE-WRIGHT, Hannah; TAYLOR, Joe. The positive impact of structured surfing courses on the wellbeing of vulnerable young people. Community Practitioner, v. 88, n. 1, p. 26-29, 2015.

KAMPION, Drew; BROWN, Bruce. Stoked-Uma história da cultura do Surf. Lisboa: Difel, 1988.

MARSHALL, Jamie; KELLY, Paul; NIVEN, Ailsa. "When I Go There, I Feel Like I Can Be Myself." Exploring Programme Theory within the Wave Project Surf Therapy Intervention. International Journal of Environmental Research and Public Health, v. 16, n. 12, p. 01-17, 2019. doi:

https://dx.doi.org/10.3390/ijerph16122159

MOHER, David; SHAMSEER, Larissa; CLARKE, Mike; GHERSI, Davina; LIBERATI, Alessandro; PETTICREW, Mark; SHEKELLE, Paul; STEWART, Lesley. Preferred reporting items for systematic review and meta-analysis protocols (PRISMA-P) 2015 statement. Systematic reviews, v. 4, n. 1, p.1-9, 2015. doi: https://dx.doi.org/10.1136/bmj.g7647

MORGAN, Paul. Get up. Stand up. Riding to resilience on a surfboard. Child and Family Social Work, v. 15, n. 1, p. 56-65. 2010. doi: https://10.1111/j.1365-2206.2009.00637.x

OLIVE, Rebeca; MCCUAIG, Luise; PHILLIPS, Murray. Women's recreational surfing: a patronising experience. Sport, Education and Society, v. 20, n. 2, p. 258-276, 2015. doi: https:

//dx.doi.org/10.1080/13573322.2012.754752

PARTINGTON, Sarah; PARTINGTON, Elisabeth; OLIVIER, Steve. The dark side of flow: A qualitative study of dependence in big wave surfing. The sport psychologist, v. 23, n. 2, p. 170-185, 2009. doi: https://dx.doi.org/10.1123/tsp.23.2.170

PÉREZ-GUTIÉRREZ, Mikel; COBO-CORRALES, Carlos. Produção científica do surfe indexada na web of science e scopus (1967-2017). Movimento v. 26, p. 1-17. 2020. doi:

https://dx.doi.org/10.22456/1982-8918.94062

PITTSINGER, Ryan; KRESS, Jeff; CRUSSEMEYER, Jill. The effect of a single bout of surfing on exercise-induced affect. International journal of exercise science, v. 10, n. 7, p. 98, 2017.

ROGERS, Carly; MALLINSON, Trudy; PEPPERS, Domonique. High-intensity sports for posttraumatic stress disorder and depression: Feasibility study of ocean therapy with veterans of Operation Enduring Freedom ocean therapy with veterans of Operation Enduring Freedom and Operation Iraqi Freedom. American Journal of Occupational Therapy, v. 68, n. 4, p. 395-400, 2014. doi: https://dx.doi.org/10.5014/ajot.2014.011221

ROY, Georgina. 'Taking emotions seriously': Feeling female and becoming-surfer through UK Surf Space. Emotion, space and society, v. 12, p. 41-48, 2014. doi: https://dx.doi.org/10.1016/j.emospa.2013.07.004

RYNNE, Steven. Exploring the pedagogical possibilities of Indigenous sport-for-development programmes using a socio-personal approach. Sport, Education and Society, v. 2, n. 4, p. 605-622, 2016. doi:

https://dx.doi.org/10.1080/13573322.2015.1107830 
SCHMID, Simone; SHORT, Cara Troy; NIGG, Claudio. Physical Activity; People with Disabilities - A Qualitative Process and Outcome Pilot Evaluation of the Non-Profit Organization AccesSurf Hawai 'i.

Hawai'i Journal of Medicine \& Public Health, v, 78, n. 2, p. 52, 2019.

SOUZA, Luciana. Pesquisa com análise qualitativa de dados: conhecendo a Análise Temática. Arquivos Brasileiros de Psicologia, v.71, n.2, p. 51-67, 2019. doi:

http://dx.doi.org/10.36482/1809-5267.ARBP2019v71i2p.51-67.

SOUZA, Marcela; SILVA, Michelly; CARVALHO, Rachel. Revisão integrativa: o que é e como fazer. Einstein (São Paulo), v. 8, n. 1, 2010. doi: https://doi.org/10.1590/s1679-45082010rw1134

SCHVIRTZ, Erica; BENSOUSSAN, Laurent; TOURRET COUDERC, Bertrand; VITON, Jean; DELARQUE, Alain; KERZONCUF, Marjiore. Return to surfing using an adapted prosthesis: A case report. Prosthetics and orthotics international, v. 42, n. 4, p. 455-459, 2018. doi: https://dx.doi.org/10.1177\%2F0309364618757785

SMITS, Froukje. Young Dutch commercially sponsored kite surfers: free as a bird? Sport in Society, v. 22, n. 10, p. 1707-1723, 2019. doi: https://dx.doi.org/10.1080/17430437.2018.1440703

THORPE, Holly. Natural disaster arrhythmia and action sports: The case of the Christchurch earthquake. International review for the sociology of sport, v. 50, n. 3, p. 301-325, 2015. doi: https://dx.doi.org/10.1177\%2F1012690213485951

THORPE, Holly. Action sports for youth development: Critical insights for the SDP community.

International journal of sport policy and politics, v. 8, n. 1, p. 91-116, 2016. doi: https://dx.doi.org/10.1080/19406940.2014.925952

WARSHAW, Matt. The history of surfing. San Francisco: Chronicle Books, 2010.

WALTER, Kristen, OTIS, Nicholas, GLASSMAN, Lisa, RAY, Travis, N., MICHALEWICZ-KRAGH, Betty;

ELLIOTT, Kim T. Kobayashi, THOMSEN, Cynthia. Comparison of surf and hike therapy for active duty service members with major depressive disorder: Study protocol for a randomized controlled trial of novel interventions in a naturalistic setting. Contemporary Clinical Trials Communications, v. 16, p. 1-10, 2019a. doi: https://doi.org/10.1016/j.conctc.2019.100435

WALTER, Kristen; OTIS, Nicholas; RAY, Travis; GLASSMAN, Lisa; MICHALEWICZ-KRAGH, Betty; POWELL, Alexandra; THOMSEN, Cynthia. Breaking the surface: Psychological outcomes among US active duty service members following a surf therapy program. Psychology of Sport and Exercise, v. 45, p. $1-9,2019$ b. doi: https://doi.org/10.1016/j.psychs port.2019.101551

WHEATON, Belinda. Surfing through the life-course: Silver surfers' negotiation of ageing. Annals of Leisure Research, v. 20, n. 1, p. 96-116, 2017. doi: https://dx.doi.org/10.1080/11745398.2016.1167610

WHEATON, Belinda. Staying 'stoked': Surfing, ageing and post-youth identities. International Review for the Sociology of Sport, v. 54, n. 4, p. 387-409, 2019. doi: https://dx.doi.org/10.1177\%2F1012690217722522

WHEATON, Belinda; ROY, Georgina; OLIVE, Rebecca. Exploring critical alternatives for youth development through lifestyle sport: Surfing and community development in Aotearoa/New Zealand. Sustainability, v. 9, n. 12, p. 2298, 2017. doi: https://dx.doi.org/10.3390/su9122298

WIERSMA, Lenny. A phenomenological investigation of the psychology of big-wave surfing at Maverick's. The Sport Psychologist, v. 28, n. 2, p. 151-163, 2014. doi: https://dx.doi.org/10.1123/tsp.2013-0001 


\section{NOTAS DE AUTOR}

\section{AGRADECIMENTOS}

Os autores agradecem à Pró-Reitoria de Extensão da Universidade Federal Fluminense (PROEX-UFF) pelo apoio ao projeto sUrFF Terapia (Sigproj 361156.2021.241704.08112020), idealizado a partir deste estudo.

\section{CONTRIBUIÇÃO DE AUTORIA}

Não se aplica.

\section{FINANCIAMENTO}

Conselho Nacional de Desenvolvimento Científico e Tecnológico (CNPq) e Pró-Reitoria de Pesquisa, Pós-Graduação e Inovação (PROPPI-UFF) - Programa Institucional de Bolsas de Iniciação Científica (IC200081).

\section{CONSENTIMENTO DE USO DE IMAGEM}

Não se aplica.

\section{APROVAÇÃO DE COMITÊ DE ÉTICA EM PESQUISA}

Não se aplica.

\section{CONFLITO DE INTERESSES}

Não se aplica.

\section{LICENÇA DE USO}

Os autores cedem à Motrivivência - ISSN 2175-8042 os direitos exclusivos de primeira publicação, com o trabalho simultaneamente licenciado sob a Licença Creative Commons Attribution Non-Comercial ShareAlike (CC BY-NC SA) 4.0 International. Esta licença permite que terceiros remixem, adaptem e criem a partir do trabalho publicado, desde que para fins não comerciais, atribuindo o devido crédito de autoria e publicação inicial neste periódico desde que adotem a mesma licença, compartilhar igual. Os autores têm autorização para assumir contratos adicionais separadamente, para distribuição não exclusiva da versão do trabalho publicada neste periódico (ex.: publicar em repositório institucional, em site pessoal, publicar uma tradução, ou como capítulo de livro), com reconhecimento de autoria e publicação inicial neste periódico, desde que para fins não comerciais e compartilhar com a mesma licença.

\section{PUBLISHER}

Universidade Federal de Santa Catarina. Programa de Pós-Graduação em Educação Física. LaboMídia - Laboratório e Observatório da Mídia Esportiva. Publicado no Portal de Periódicos UFSC. As ideias expressadas neste artigo são de responsabilidade de seus autores, não representando, necessariamente, a opinião dos editores ou da universidade.

\section{EDITORES}

Mauricio Roberto da Silva, Giovani De Lorenzi Pires, Rogério Santos Pereira.

\section{EDITOR DE SEÇÃO}

Rogério Santos Pereira. 


\section{REVISÃO DO MANUSCRITO E METADADOS}

João Caetano Prates Rocha; Keli Barreto.

\section{HISTÓRICO}

Recebido em: 23 de maio de 2020.

Aprovado em: 27 de janeiro de 2021.

\section{APÊNDICES}

Tabela 1 - Dados gerais dos artigos incluídos na revisão com os descritores "Surf” AND "Psychology”.

\begin{tabular}{|c|c|c|c|c|}
\hline Artigo & Objetivos & $\begin{array}{c}\text { Características } \\
\text { amostrais }\end{array}$ & Instrumentos & Resultados \\
\hline $\begin{array}{l}\text { Correia e } \\
\text { Rosado (2019) }\end{array}$ & $\begin{array}{l}\text { Investigar } \\
\text { diferenças de } \\
\text { gênero quanto à } \\
\text { ansiedade. }\end{array}$ & $\begin{array}{l}601 \text { atletas ( } 172 \\
\text { mulheres e } 429 \\
\text { homens). }\end{array}$ & $\begin{array}{l}\text { Escala de } \\
\text { ansiedade } \\
\text { esportiva } \\
\text { (SAS-2); } \\
\text { questionário }\end{array}$ & $\begin{array}{l}\text { Maiores níveis } \\
\text { de ansiedade em } \\
\text { mulheres e em } \\
\text { esportes } \\
\text { individuais. }\end{array}$ \\
\hline $\begin{array}{l}\text { Pittsinger et al. } \\
\text { (2017) }\end{array}$ & $\begin{array}{l}\text { Verificar efeitos } \\
\text { de uma única } \\
\text { sessão de surfe } \\
\text { sobre o Afeto } \\
\text { induzido pelo } \\
\text { exercício. }\end{array}$ & $\begin{array}{l}107 \text { adultos } \\
\text { praticantes de } \\
\text { surfe (91 } \\
\text { homens e } 16 \\
\text { mulheres). }\end{array}$ & $\begin{array}{l}\text { Questionário } \\
\text { Demográfico; } \\
\text { Escala de } \\
\text { Afetividade } \\
\text { (PAAS). }\end{array}$ & $\begin{array}{l}\text { Aumento do } \\
\text { afeto positivo e } \\
\text { da tranquilidade; } \\
\text { diminuição do } \\
\text { afeto negativo. }\end{array}$ \\
\hline Wiersma (2014) & $\begin{array}{l}\text { Estudar aspectos } \\
\text { psicológicos na } \\
\text { competição de } \\
\text { surf de ondas } \\
\text { grandes. }\end{array}$ & 7 competidores. & $\begin{array}{l}\text { Entrevistas } \\
\text { fenomenológicas. }\end{array}$ & $\begin{array}{l}\text { Variação } \\
\text { emocional } \\
\text { conforme } \\
\text { momentos da } \\
\text { competição. }\end{array}$ \\
\hline $\begin{array}{l}\text { Partington et al. } \\
(2009)\end{array}$ & $\begin{array}{l}\text { Estudar aspectos } \\
\text { negativos do } \\
\text { surfe. }\end{array}$ & $\begin{array}{l}15 \text { atletas de } \\
\text { elite no surfe } \\
\text { competitivo. }\end{array}$ & $\begin{array}{l}\text { Entrevista } \\
\text { semiestruturada. }\end{array}$ & $\begin{array}{l}\text { Indícios de } \\
\text { dependência } \\
\text { psicológica em } \\
\text { praticantes. }\end{array}$ \\
\hline $\begin{array}{l}\text { Brymer e Oades } \\
(2009)\end{array}$ & $\begin{array}{l}\text { Investigar a } \\
\text { aprendizagem da } \\
\text { humildade e da } \\
\text { coragem em } \\
\text { esportes de risco. }\end{array}$ & $\begin{array}{l}15 \text { atletas de } \\
\text { esportes esportes } \\
\text { radicais. }\end{array}$ & $\begin{array}{l}\text { Entrevistas } \\
\text { fenomenológicas. }\end{array}$ & $\begin{array}{l}\text { Humildade e } \\
\text { coragem podem } \\
\text { ser adquiridas } \\
\text { com a prática de } \\
\text { esportes } \\
\text { radicais. }\end{array}$ \\
\hline
\end{tabular}

Legenda: SAS (Sport Anxiety Scale); PAAS (Physical Activity Affect Scale)

Tabela 2 - Dados gerais dos artigos incluídos na revisão com os descritores "Surf" AND "Sport”.

\begin{tabular}{lllll}
\hline Artigo & Objetivos & Amostra & Instrumentos & Resultados \\
\hline \multirow{3}{*}{$\begin{array}{l}\text { Wheaton } \\
(2019)\end{array}$} & Estudar & 11 surfistas, de 45 & entrevistas & O surfe \\
& surfe de lazer & a 70 anos. & qualitativas. & desencadeou \\
& em surfistas & & & $\begin{array}{l}\text { e facilos positivos } \\
\text { engajamento nas }\end{array}$
\end{tabular}


idosos e de

meia-idade.

Schmid et al Avaliar efeitos (2019)

Burgess et al (2019)

Evers (2019)
Avaliar a
dimensão emocional do trabalho como 'freesurfers" patrocinados para divulgação digital.

\section{Schvirtz et al. (2018)}

Wheaton et al. (2017)

Wheaton (2017)

Caddick e Smith (2017)

\author{
Verificar se os \\ sintomas de \\ TEPT e \\ ansiedade de um \\ amputado \\ transfemural são \\ influenciados \\ pela prática do \\ surf. \\ Estudar efeitos \\ de programas de \\ desenvolviment \\ o social através \\ do surfe.

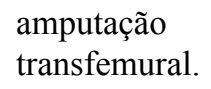 \\ Voluntários de 8 \\ programas de \\ desenvolvimento \\ social na Nova \\ Zelândia.
}

Explorar
significados do
surf recreacional
nas vidas e
identidades dos
participantes à
medida que
envelhecem.

Investigar se a prática do surfe

6 participantes (4
com Lesão
Medular e 2 com
quadros
neurológicos).

227 Surfistas registrados no Australian Surfing Titles (2014).

\section{1 surfistas profissionais .}

11 surfistas que começaram a surfar após os 40 anos.

15 veteranos de guerra e 1
Análise do discurso

Formulário online.

Entrevistas semiestruturadas

Entrevista.

Levantamento
sobre
características
dos projetos de
surf; entrevistas.

Entrevistas e técnicas de observação.

Entrevistas semiestruturadas. pessoas idosas e

de meia idade.

Efeitos positivos

do programa

para as esferas

física,

psicológica e

social.

Indicadores de

saúde física e

mental são

maiores em

surfistas do que

em amostra

normativa.

O trabalho como

freesurfer

apresenta

peculiaridades

indutoras de

tensão, cobrança

e expectativas

que podem afetar

o bem estar

emocional.

Diminuição da

ansiedade,

melhoria na

qualidade de

vida, bem-estar e

diminuição de

sintomas de

TEPT.

Observou-se

melhoria nos

indicadores

sociais, aumento

do bem-estar e

da capacidade de autorregulação.

Foram

identificadas

sensações de

euforia;

desenvolvimento

da

espiritualidade; surfe como fator constituinte da identidade.

Diminuição dos sintomas de 
pode auxiliar

veteranos de

guerra com

sintomas de

TEPT e

depressão.

$\begin{array}{ll}\text { Amrhein et } & \text { Efeitos do surfe } \\ \text { al. (2016) } & \begin{array}{l}\text { em sintomas de } \\ \text { depressão e de } \\ \text { ansiedade. }\end{array}\end{array}$

Bush (2016)

Estudar a cultura de mulheres

surfistas de uma comunidade da costa leste australiana.

$\begin{array}{ll}\text { Rynne } & \text { Investigar o } \\ \text { (2016) } & \text { papel do surfe } \\ & \text { no } \\ & \text { desenvolviment } \\ & \text { o físico e social } \\ \text { de adolescentes } & \text { indígenas. }\end{array}$

Brasil et al. Estudar ações (2016)

Furley e Dor (2016)

Thorpe

(2015)

adotadas por professores de surfe. ex-integrante de serviços civis.

100 surfistas.

.

20 surfistas

54 indígenas.

5 instrutores de surfe.

$\begin{array}{ll}\begin{array}{l}\text { Pesquisar se as } \\ \text { habilidades } \\ \text { decisórias } \\ \text { variam em } \\ \text { função da } \\ \text { experiência no } \\ \text { surfe. }\end{array} & \begin{array}{l}\text { 76 participantes } \\ \text { com diferentes } \\ \text { níveis de } \\ \text { experiência. }\end{array} \\ \begin{array}{l}\text { Avaliar efeitos } \\ \text { terapêuticos dos } \\ \text { esportes de ação } \\ \text { para minimizar }\end{array} & \begin{array}{l}\text { 14 atletas de } \\ \text { esportes de ação } \\ \text { variados que } \\ \text { vivenciaram o } \\ \text { terremoto de }\end{array}\end{array}$

TEPT; bem-estar e maior percepção de liberdade.

\begin{abstract}
Questionário demográfico, questionário específico sobre experiência espiritual; escala de avaliação da espiritualidade, Inventário de depressão e de ansiedade.
\end{abstract}

Observação
participante;
entrevistas
semi-estruturada
s e questionário
online.

Notas de campo, fotografias, técnicas de observação, análises documentais; entrevistas semi-estruturada s.

\section{Entrevistas semi-estruturada} s.

Tarefa de decisão em ambiente de surfe virtual.

Entrevistas
semiestruturadas.

Surfistas apresentaram menos sintomas de depressão e de ansiedade do que amostra normativa; indícios de experiências espirituais promovidas pelo surfe.

Surfe como
potencial
terapêutico,
capaz de
estimular
percepções de
felicidade.

Desenvolviment o de laços sociais; reconexão com a espiritualidade e natureza através do contato com o mar.

Instrutores que
consideram os
aspectos
psicológicos em
sua didática,
garantem maior
adesão e
propiciam mais
aprendizagem.
Melhores
decisões foram
verificadas em
surfistas mais
experientes.

A prática
esportiva parece
ter ajudado a
diminuir
sintomas de


impactos de um desastre natural.

Christchurch

(NZ), em 2011.

$\begin{array}{ll}\text { Godfrey et } & \text { Verificar se } \\ \text { al. (2015) } & \text { projeto social } \\ & \text { com surfe } \\ & \text { influencia a } \\ & \text { sociabilidade de } \\ & \text { jovens. }\end{array}$

Olive et al.

(2015)

Caddick et

al. (2015a)

Caddick, Smith e Phoenix (2015b)

Beaumont e Brown (2015)

\section{Estudar} experiências de mulheres no surfe.
15 veteranos homens e com

baseada na natureza, no bem-estar dos veteranos de guerra diagnosticados com TEPT.

\author{
114 jovens (oito a \\ 18 anos) com \\ problemas de \\ saúde mental ou \\ exclusão social.
}

11 surfistas mulheres.

15 veteranos de guerra.

prática do surfe influenciou a vida de veteranos de guerra. idades que variam de 27 a 60 anos.

Verificar
influências do
surfe em uma
comunidade da

29 surfistas de uma aldeia situada no sudoeste da Inglaterra.
Escala de

Bem-estar para

Crianças

(SCWBS) e entrevista com pais.

Entrevistas.

Entrevistas

semiestruturadas.

estresse,

ansiedade e níveis de tensão; Aumentando capacidades de da resiliência.

Maiores índices de felicidade (98\% da amostra); maior sociabilidade (89\% da amostra).

Surfe foi associado a sensações de prazer, empolgação e bem-estar; aumento do sentimento de pertencimento à comunidade aspectos negativos: marginalização e sexualização

Surfe ajudou a resignificar valores militares rígidos; melhorou indicadores de saúde mental; aumentou bem-estar e diminuiu sintomas de TEPT.

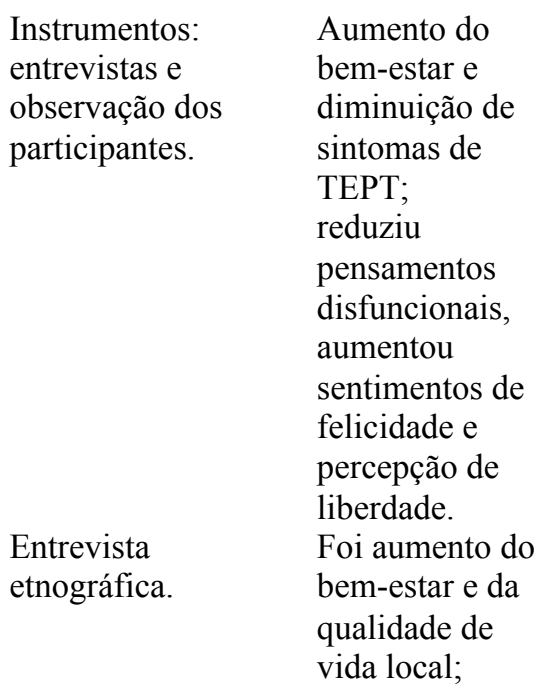


Cornalina, Inglaterra.

Thorpe

(2016)

$\begin{array}{ll}\text { Roy (2014) } & \text { Estudo sobre o } \\ & \text { significado do } \\ & \text { afeto e das } \\ & \text { emoções no } \\ & \text { processo de } \\ & \text { formação no } \\ & \text { surfe feminino. }\end{array}$

Rogers et al. (2014)

Investigou o
potencial
terapêutico do
surfe e efeitos
psicossociais em
veteranos de
guerra com
TEPT.

Marshall et Investigar al. (2019)
Investigar novas tendências na participação de jovens em esportes de ação e o potencial destas modalidades para promoção da paz.
Praticantes de esportes de ação não institucionalizados . 35 mulheres com
idades entre 12 e 65
anos.

11 veteranos de guerra com sintomas de TEPT e de depressão maior.

22 participantes. indícios de

Desenvolviment

o financeiro e da comunidade de uma forma geral.

Foram

evidenciados

efeitos destas modalidades no desenvolvimento de valores morais, criatividade e suas implicações para políticas públicas e promoção da paz.

Entrevistas semiestruturadas.

Apesar do domínio masculino, a fluidez do espaço permite o empoderamento feminino e a experiência de liberdade.

Questionários de auto-relato.

Surfe diminuiu sintomas de TEPT e de depressão. Aumentou sentimentos de confiança.

Surfe
desencadeou
maior de
autonomia,
bem-estar,
confiança,
maiores índices
de resiliência, e
outras melhorias
físicas e sociais.

Surfe Entrevistas. projeto social com surfe na saúde mental de jovens. 